\title{
An lterative Procedure for Linking Metrics and Assessing Item Bias in Item Response Theory
}

\author{
Gregory L. Candell and Fritz Drasgow

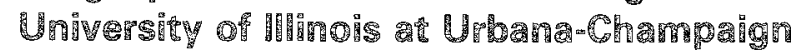

\begin{abstract}
The presence of biased items may seriously affect methods used to link metrics in item response theory. An iterative procedure designed to minimize this methodological problem was examined in a monte carlo investigation using the two-parameter item response model. The iterative procedure links the scales of independently calibrated parameter estimates using only those items identified as unbiased. Two methods for transforming parameter estimates to a common merric were incorporated into the iterative procedure. The first method links scales by equating the first two moments of the distributions of estimated item difficulties. The second method determines the linking transformation by minimizing differences across IRT characteristic curve estimates. Results indicate that iterative linking provides a substantial improvement in item bias detection over the noniterative approach. Index terms: Item bias, Item response theory, Iterative method, Linking, Metric linking, Two-parameter item response model.
\end{abstract}

Applications of item response theory (IRT) requiring a common metric for parameter estimates may be seriously affected by the presence of biased items. Examinations of item bias, for example, compare item parameter estimates across groups and therefore may be susceptible to this methodological artifact. Accurate linking of item parameters is required for meaningful comparisons. Errors in the linking transformation may result in

APPLIED PSYCHOLOGLCAL MEASUREMENT

Vol. 12, No. 3, September 1988, pp. 253-260

(C) Copyright 1988 Applied Psychological Measurement Inc. 0146-6216/88/030253-08\$1.65 spurious instances of item bias (Shepard, Camilli, \& Williams, 1984). Items truly containing substantial bias may lead to such errors, degrading the uransformation of scale and adversely affecting subsequent item bias analyses.

Lord (1980, pp. 220-221) outlined a "purification" strategy, first suggested by Marco (1977), for reducing the potential effects of biased items on parameter estimation and subsequent item bias analyses. The procedure involves initial item parameter estimation, identification of item bias, estimation of abilities based on those items identified as unbiased, and reestimation of item parameters by fixing ability estimates at those values obtained with the unbiased items. The rationale for this approach is that biased items will degrade ability estimation, which in turn may adversely affect item bias identification (McCauley \& Mendoza, 1985).

This article uses an alternative procedure first suggested by Segall (1983). It is easier to implement than Marco's method in that reestimation of ability and item parameters is not required. The procedure involves the following steps: (1) initial linking of metrics across independently calibrated groups, (2) calculating a bias index for each item, (3) relinking group metrics using only those items identified as unbiased in step 2, and (4) recalculating bias indices for all items. Steps 3 and 4 are repeated until the same items are identified as biased for two successive iterations. The final assessment of item bias is based on a transformation that uses 
only items previously identified as unbiased. This approach to item bias detection is similar in spirit to the iterative logit method used by van der Flier and associates (Kok, Mellenbergh, \& van der Flier, 1985; van der Flier, Mellenbergh, Ader, \& Wijn, 1984) in that items identified as biased at each iteration are excluded from the initial computations in the subsequent iteration.

Iterative linking has been used in studies of measurement bias (Drasgow, 1987) and translation bias (Candell \& Hulin, 1986; Hulin \& Mayer, 1986) and may offer a substantial improvement in IRT linking and item bias detection over the conventional, noniterative approach. An examination of the relative contributions of iterative and noniterative linking to the detection of item bias is needed to determine the efficacy of the iterative approach. The present study addresses this need, using monte carlo methods to conpare the recovery of simulated item bias across iterative and noniterative procedures.

\section{Methender}

\section{Latenc}

The latent trait $(\theta)$ distributions for the simulated examinee groups remained constant throughout the siudy. Os were sampled from a normal $(0,1)$ distribution for the "base" group (Lim, Levine, Hastings, \& Wardrop, 1981), while "comparison" group parameters were also sampled from a normal $(0,1)$ distribution and transformed to have a mean of -1.00 and variance of 1.00 . This discrepancy across $\theta$ distributions would appear to be a good test for the linking procedures used in this study, representing neither a trivial nor an extreme situation, but rather one with potentially serious consequences that may often be encountered in practice.

\section{Dasta Gereration}

Item responses were generated using the twoparameter logistic item response model. In this model, the probability of a simulated examinee giving the keyed response to the ith irem, given unidimensional latent trait $\theta$, is given as

$\mathbb{P}_{i}(\theta)=1 /\left\{1+\exp \left\{D a_{i}\left(\theta-b_{i}\right)\right]\right\}$,

where the values $a_{i}$ and $b_{i}$ are the item discrimination and item difficulty parameters, respectively, and $D$ is a scaling constant equal to -1.702 .

Dichotomous item responses were generated by computing the probability of a correct response for each item and each examinee using Equation 1. The probability of a correct response was compared to a number sampled from the $(0,1)$ uniform distribution. If the probability was less than the random number, the item response was scored 0 ; otherwise, the item response was scored 1.

\section{Iterens}

Item parameters used to generate responses were selected in a way that would make simulation results more generalizable to "real" data. The selection procedure involved plotting pairs of $a$ and $b$ estimates obtained from a previous study (Candell \& Hulin, 1986) that used the two-parameter normal ogive model, and then selecting 30 points that clustered in the most densely populated region of the plot. An additional 10 points located outside this region were also selected to represent moderate to extreme values. Table 1 lists the 40 pairs of $a$ and $b$ estimates used in generating item responses.

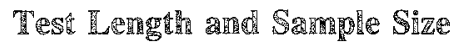

Three test lengths were examined: $n=10,20$, and 40 . Items 1 through 10 were used for the 10 item tests; items $\mathbb{1}$ through 20 were used for the 20-item tests. Sample sizes of $N=300$ and 500 were used in conjunction with the three test lengths. These sample sizes were chosen because they span the range that could be expected for large attitude surveys and, in the case of $N=500$, include the minimum sample size recommended for estimating two-parameter logistic models (Hulin, Lissak, \& Drasgow, 1982). It was considered important in the present study to determine how well the procedures used to detect biased items performed when sample sizes fell below the recommended minimum for parameter estimation. 
Table 1

Item Parameters Used

to Generate

Response Data

\begin{tabular}{ccc} 
Iten & 2 & 8 \\
\hline 1 & .90 & -1.23 \\
2 & .94 & -.18
\end{tabular}

$1.16-1.06$

$.88 \quad-.92$

$.97-.71$

$.72 \quad .42$

$.37 \quad .77$

$.86 \quad 1.53$

$1.05 \quad-.06$

$1.07 \quad-.54$

$1.04-.02$

$1.48-1.24$

$.91--.34$

$1.09-1.15$

$1.71-1.42$

$1.64 \quad-.86$

$1.50-1.29$

$1.48 \quad-.43$

$.94 \quad-.27$

$1.44-.91$

$.85-.55$

$\begin{array}{ll}.51- & -.24\end{array}$

$1.12-.85$

$1.15-.99$

$.81-.62$

$1.00-1.19$

$\begin{array}{ll}.97 & -.72\end{array}$

$1.37 \quad .28$

$\begin{array}{ll}1.36 & 0.00\end{array}$

$1.50 \quad-.17$

$.90 \quad-.22$

$.44 \quad-.47$

$.76-.42$

$.31 \quad 1.62$

$.45 \quad-.89$

$1.59 \quad .29$

$1.59 \quad .82$

$.91 \quad .73$

$1.48 \quad .67$

$.75 \quad-.24$

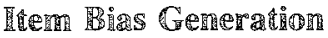

Two levels of item bias were examined. In each test, either $10 \%$ or $30 \%$ of the items were biased. ltems were made to be biased by increasing their difficulty parameters by .75 prior to generating item responses for the comparison group examinees. Table 2 provides a summary of the 12 datasets generated.

\section{Estirnation of If}

Drasgow's (in press) program for computing marginal maximum likelihood estimates (Bock \& Lieberman, 1970) was used to estimate item parameters for the two-parameter normal ogive model,

$P_{i}(0)=\int_{-\infty}^{g_{i}} \phi(t) \partial t$

where $\phi(t)$ is the nomal probability density at $t$ and $g_{i}$ is the upper limit of the integral

$g_{i}=a_{i}\left(\theta-b_{i}\right)$.

Two-parameter normal ogives and logistic ogives are nearly identical (Birnbaum, 1968) and were used interchangeably.

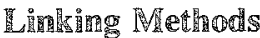

Many methods exist for transforming IRT parameter estimates from independent calibrations to a common scale. These methods, though differing in detail, allow for the equations

$$
\begin{aligned}
& \hat{\theta}(\text { base })=A \hat{\theta}(\text { comparison })+B, \\
& \hat{a}(\text { base })=\hat{a}(\text { comparison }) / A, \\
& \text { and } \\
& \hat{b}(\text { base })=A \hat{b} \text { (comparison })+B,
\end{aligned}
$$

where $A$ and $B$ are the coefficients of the linear transformation that maps the metric of the comparison group to that of the base group.

\begin{tabular}{cccc}
\multicolumn{5}{c}{$\begin{array}{c}\text { Table } 2 \\
\text { Sumary of Dataset Conditions }\end{array}$} \\
\hline $\begin{array}{c}\text { Number } \\
\text { Condition }\end{array}$ & $\begin{array}{c}\text { Percentage } \\
\text { of Items }\end{array}$ \\
\hline 1 & Size & in Scems & in Scale \\
in Scale & Biased \\
2 & 300 & 10 & 10 \\
3 & 300 & 10 & 30 \\
4 & 300 & 20 & 10 \\
5 & 300 & 20 & 30 \\
6 & 300 & 40 & 10 \\
7 & 300 & 40 & 30 \\
8 & 500 & 10 & 10 \\
9 & 500 & 10 & 30 \\
10 & 500 & 20 & 10 \\
11 & 500 & 20 & 30 \\
12 & 500 & 40 & 10 \\
\hline
\end{tabular}


Current linking methods can be classified into two groups. One class focuses on the first two moments of the distribution of $\hat{b} s$ or $\hat{\theta} s$ in each group (Bejar \& Wingersky, 1981; Linn et al., 1981; Vale, Maurelli, Gialluca, Weiss, \& Ree, 1981). These methods determine the linking transformation that will equate the means and standard deviations of the $\hat{b} s$ or $\hat{\theta}$. Poorly estimated $b s$ or $\theta \mathrm{s}$ are handled by giving less weight to these values during estimation of the moments. Linn et al. (1981), for example, computed sample moments after weighting each $\hat{b}$ by the inverse of its estimated standard error.

A second class of methods links metrics by determining the transformation that minimizes differences between item response functions, or indices obtained from these functions, across groups (Divgi, 1985; Haebara, 1980; Stocking \& Lord, 1983). The Stocking and Lord (1983) "characteristic curve" method, for example, determines the transformation of scale by minimizing differences for true score estimates (the sum of the item response probabilities for all test items) across groups.

The characteristic curve linking method would seem to represent an improvement over methods that focus only on estimated item difficulties, because the former uses more information contained in the item response function. To investigate this proposition, both the Lim et al. (1981) weighted b linking and Stocking and Lord (1983) characteristic curve linking were used within iterative and noniterative procedures. Because the marginal item parameter estimation procedure used in this study does not provide $\theta$ estimates, the 99 centile points of the standard normal distribution were used in place of estimated os when performing characteristic curve linking.

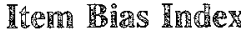

Item bias was assessed using Lord's (1980, chap. 14) $\chi^{2}$ index, a statistic that simultaneously tests the hypothesis that $a$ and $b$ are identical across groups. Standard errors and sampling covariance estimates required to compute the $\chi^{2}$ index were provided by Drasgow's (in press) program. An al- pha level of $.005\left(\chi^{2}=10.60\right)$ was used to classify items as biased during the iterative linking procedure.

\section{Resals}

\section{具世em 路ỉs Recovery}

The relative efficacy of iterative and noniterative linking procedures in distinguishing biased items from unbiased items was evaluated by computing the median and largest $\chi^{2}$ values for biased and unbiased items for each of the 12 conditions. Each condition contained 5 independent replications to reduce the effect of sampling variability and ensure more stable results. Table 3 lists the results for each linking method, averaged across the five replications, for both noniterative and iterative procedures. These results are meaningful only when evaluated within conditions, because the same set of items was not tampered with across conditions; aggregate values obtained for different sets of items are not comparable. The number of iterations required for iterative solutions ranged from 2 to 6 .

Table 3 shows that, for each of the 12 conditions and both the characteristic curve and weighted $\hat{b}$ linking methods, iterative linking provided improved bias detection when applied to the same item parameter estimates used with the noniterative approach. Iterative linking increased the median and largest $\chi^{2}$ values for truly biased items, while these values decreased for unbiased items. For sample sizes of 500 (conditions 7 through 12) the improvement was substantial. For example, median and largest $\chi^{2}$ values for condition 12 ( $n=$ $40, N=500,30 \%$ biased items), using noniterative characteristic curve linking, were 20.72 and 45.71 , respectively. Application of iterative characteristic curve linking increased the median and largest values to 38.86 and 83.20 , respectively. At the same time, the median $\chi^{2}$ for unbiased items decreased from 6.29 to 1.68 , and the largest $\chi^{2}$ among unbiased items decreased from 24.07 to 13.51 , when the iterative procedure was used.

Results for conditions 1 through 6 are similar to those of conditions 7 through 12 , though not as 
Table 3

Median and Largest $\chi^{2}$ Values for Noniterative (N) and Iterative (I)

procedures, for the Weighted $\hat{b}$ and Characteristic Curve Linking Methods (Weans Over 5 Replications)

\begin{tabular}{|c|c|c|c|c|c|c|c|c|}
\hline \multirow[b]{3}{*}{ Condition } & \multicolumn{4}{|c|}{ Unbiased Items } & \multicolumn{4}{|c|}{ Biased Itens } \\
\hline & \multicolumn{2}{|c|}{ Median } & \multicolumn{2}{|c|}{ Largest } & \multicolumn{2}{|c|}{ Median } & \multicolumn{2}{|c|}{ Largest } \\
\hline & $\mathrm{N}$ & $I$ & IV & I & H & I & $\mathrm{N}$ & I \\
\hline \multicolumn{9}{|c|}{ Weighted $\hat{b}$ Methoo } \\
\hline $1^{6}$ & 1. 33 & 1.00 & 5.62 & 4.08 & 12.99 & 21.62 & 12.99 & 21.62 \\
\hline 2 & 1.75 & 1.34 & 5.14 & 4.10 & 8.71 & 9.96 & 14.59 & 16.98 \\
\hline 3 & 1.16 & 1.07 & 7.67 & 7.62 & 11.89 & 13.26 & 17.16 & 19.22 \\
\hline 4 & 1.68 & 1.19 & 7.06 & 4.77 & 9.69 & 12.41 & 20.62 & 24.98 \\
\hline 5 & 1.78 & 1.68 & 10.43 & 9.50 & 18.44 & 20.33 & 28.55 & 31.11 \\
\hline 6 & 3.09 & 1.62 & 16.26 & 9.73 & 16.13 & 24.61 & 43.11 & 63.58 \\
\hline 7 & 1.87 & 1.28 & 6.20 & 4.43 & 23.95 & 30.22 & 23.95 & 30.22 \\
\hline 8 & 2.16 & 1.14 & 8.47 & 4.38 & 13.21 & 19.96 & 19.53 & 26.70 \\
\hline 9 & 1.40 & 1.31 & 5.86 & 5.24 & 20.18 & 22.41 & 29.40 & 32.63 \\
\hline 10 & 2.45 & .84 & 7.70 & 4.84 & 21.43 & 29.35 & 35.30 & 47.83 \\
\hline 11 & 1.99 & 1.93 & 9.38 & 8.02 & 28.28 & 32.09 & 38.39 & 43.03 \\
\hline 12 & 4.07 & 1.57 & 18.50 & 13.65 & 25.90 & 41.01 & 54.38 & 87.08 \\
\hline \multicolumn{9}{|c|}{ Characteristic Curve Method } \\
\hline $1^{b}$ & 1.38 & 1.23 & 4.92 & 4.06 & 12.24 & 18.78 & 12.24 & 18.78 \\
\hline $2^{\circ}$ & 2.19 & 1.44 & 8.76 & 7.98 & 5.37 & 6.93 & 12.44 & 15.64 \\
\hline 3 & 1.38 & 1.16 & 7.58 & 7.12 & 10.57 & 11.84 & 15.17 & 17.04 \\
\hline $4^{\circ}$ & 2.60 & 1.32 & 10.39 & 7.15 & 7.90 & 11.38 & 17.25 & 24.83 \\
\hline 5 & 2.00 & 1.76 & 11.89 & 9.72 & 16.43 & 18.98 & 26.19 & 29.41 \\
\hline 6 & 4.52 & 2.18 & 21.92 & 11.99 & 13.05 & 21.34 & 36.67 & 57.72 \\
\hline 7 & 1.56 & 1.35 & 8.51 & 5.14 & 22.19 & 28.81 & 22.19 & 28.81 \\
\hline 8 & 3.21 & 1.71 & 12.85 & 7.31 & 9.85 & 16.27 & 15.20 & 22.36 \\
\hline 9 & 1.68 & 1.31 & 6.74 & 5.57 & 18.17 & 21.07 & 26.56 & 30.76 \\
\hline 10 & 4.30 & 1.17 & 11.06 & 5.57 & 16.51 & 27.07 & 28.23 & 44.53 \\
\hline 11 & 2.04 & 1.95 & 11.50 & 8.54 & 25.67 & 30.79 & 35.04 & $41 \cdot 34$ \\
\hline 1.2 & 6.29 & 1.68 & 24.07 & 13.51 & 20.72 & 38.86 & 45.71 & 83.20 \\
\hline
\end{tabular}

Three of the 5 replications for this condition did not yield any initial $x^{2}$ values above the critical value of 10.60 , and the iterative procedure was not invoked. The iterative results reported here are means over the other 2 replications.

Two of the 5 replications for this condition did not yield any initial $x^{2}$ values above the critical value of 10.60 , and the iterative procedure was not invoked. The iterative results reported here are means over the other 3 replications.

'One of the 5 replications for this corrdition did not yield any initial $x^{2}$ values above the critical value of 10.60 , and the lterative procedure was not invoked. The iterative results reported here are means over the other 4 replications.

impressive. Samples of 300 were insufficient to generate the necessary statistical power to detect several of the biased items in this study. The inability to identify these items decreased the effectiveness of the iterative procedure, though iterative linking still improved item bias recovery when compared to the noniterative approach.

Figure 1 demonstrates graphically the improved scale transformation resulting from iterative linking. Estimated item difficulty parameters for the base group, obtained from one replication of condition 12 using weighted $\hat{b}$ linking, are plotted against corresponding comparison group estimates. The diagonal line in Figures $1 \mathrm{a}$ and $1 \mathrm{~b}$ represents the transformation $A=1.00$ and $B=-1.00$ in Equations 4 through 6 that places the comparison group estimates on the base group metric. Items simulated to be biased are indicated by open circles. After iterative linking, the increased clustering of unbiased items along the diagonal is evident. 
Figune 1

Base Group Estimated Item Difficulties Plotted Against

Comparison Group Estimared Item Difficulties

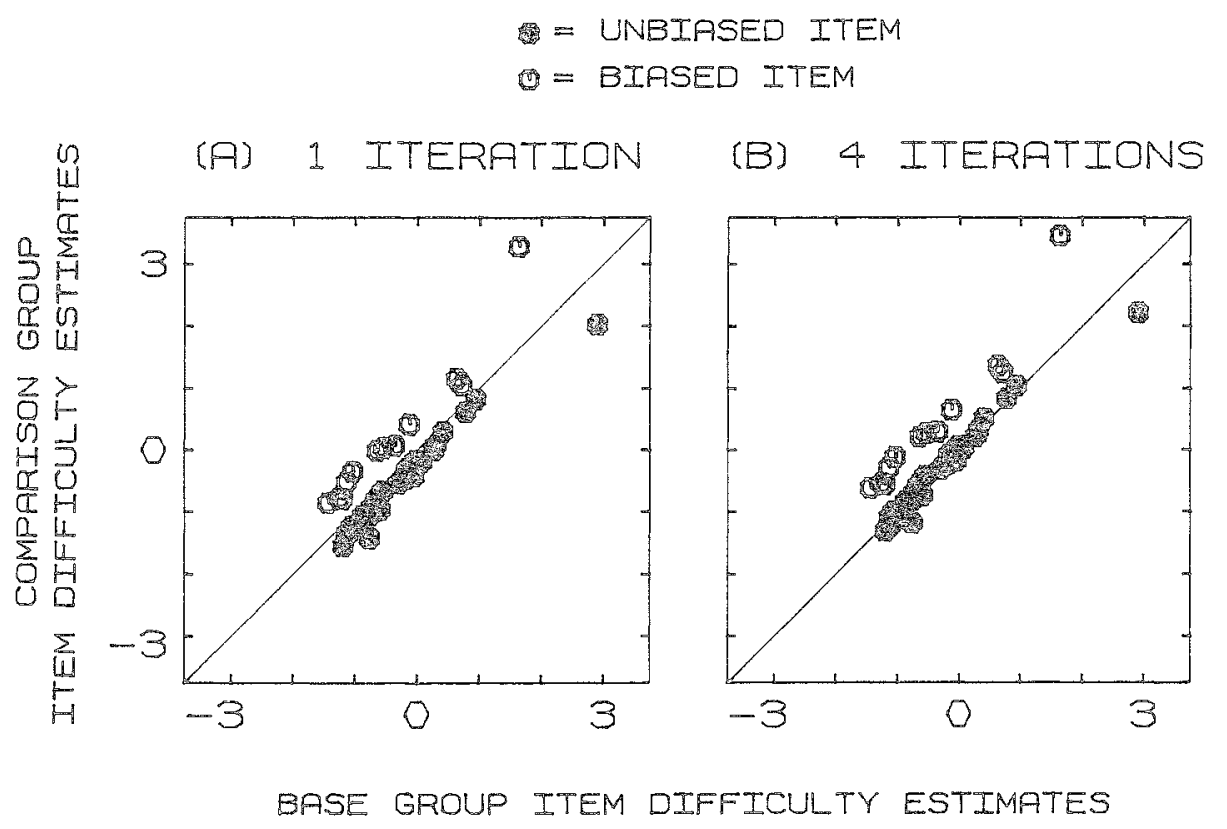

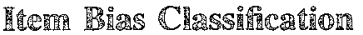

Improved differentiation of biased and umbiased items provided by iterative linking should also result in differences in final bias classification when using a specified critical value for the bias index. $\chi^{2}$ values for some truly biased items initially idenuified as unbiased may exceed the crirical value for significance after iterating; "hit" rates will increase. Conversely, unbiased items with $\chi^{2}$ values initially exceeding the critical value may be reclassified as unbiased; "false alarm" rates will decrease. Table 4 shows the mean hit and false alarm rates for moniterative and iterative linking, using a critical $x^{2}$ of $10.60(p<.005)$. Conditions 4 through 6 and 10 through 12 were chosen for this analysis because they contain sufficient numbers of biased items to provide meaningful comparisons. The same warning for interpreting Table 3 applies to Table 4; only comparisons within conditions are appropriate.
The results reported in Table 4 demonstrate the significant impact of biased items on metric linking and bias classification. Condition $12(N=500$, $n=40$, and $30 \%$ biased items), using the characteristic curve linking method, provides the mosi dramatic example. Noniterative linking yielded a mean hit rate of .78 and a mean false alarm rate of .26. That is, an average of 9 of the 12 truly biased items were correctly identified while an ayerage of 7 of the 28 unbiased items were incorrectly classified as biased. Iterative linking increased the mean hit rate to .88 and reduced the mean fallse alarm rate to .03 . Thus, the linking transformation obtained after iterating decreased the incidence of false alarms from an average of 7 items to an average of 1 irem among the 28 unbiased items.

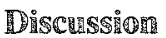

For IRT applications such as item bias analyses, the utility of a linking procedure rests in its ability 
Table 4

Mean Hic and False Alarn (FA) Rates

for Item Blas Identification, for

Noniterative and Iterative Procedures Using the Welghted $\hat{b}$ and the

Characteristic Curve Linking kethods

\begin{tabular}{ccccc}
\hline & \multicolumn{2}{c}{ Noniterative } & \multicolumn{2}{c}{ Iterative } \\
\cline { 2 - 3 } Condition & $\begin{array}{c}\text { Mit } \\
\text { Rate }\end{array}$ & $\begin{array}{c}\text { RA } \\
\text { Rate }\end{array}$ & $\begin{array}{l}\text { Hit } \\
\text { Rate }\end{array}$ & $\begin{array}{c}\text { FA } \\
\text { Rate }\end{array}$ \\
\hline $\begin{array}{c}\text { Weighted } \hat{b} \\
4\end{array}$ & .40 & .00 & .50 & .00 \\
5 & .75 & .01 & .75 & .01 \\
6 & .70 & .10 & .80 & .02 \\
10 & .70 & .00 & .73 & .00 \\
11 & .85 & .01 & .85 & .01 \\
12 & .82 & .11 & .90 & .03 \\
Characteristic Curve Method & & \\
4 & .33 & .03 & .50 & .01 \\
5 & .65 & .02 & .75 & .01 \\
6 & .62 & .16 & .77 & .04 \\
10 & .67 & .08 & .70 & .00 \\
11 & .80 & .02 & .85 & .01 \\
12 & .78 & .26 & .88 & .03 \\
\hline
\end{tabular}

to provide the linking transformation resulting in the most accurate assessment of item bias. The findings reported in this study indicate that iterative linking provides improved bias detection over the conventional noniterative approach. Application of the iterative procedure resulted in increased $\chi^{2}$ vallues for trully biased items and decreased $\chi^{2}$ values for unbiased items. More importantly, increased differentiation of $\chi^{2}$ values between biased and unbiased items improved the final classifications of biased and unbiased items.

For the conditions used in this study, weighted $\hat{b}$ linking consistently provided the more accurate linking transformation, for both noniterative and iterative conditions, as reflected in item bias recovery. These results are surprising, because characteristic curve linking uses more information from the item response function and because the bias simulation in this study involved tampering with the $b$ parameter for each item designated as biased. One possible explanation for these findings would be that the small samples used in this study did not allow for adequare estimation of $a$ parameters. The inclusion of these poorly estimated as in the determination of the linking transformation would degrade rather than improve the accuracy of the transformation. Divgi (1985) has suggested a linking method, based on Lord's $\chi^{2}$ item bias statistic, that takes into account the standard errors of the $a$ and $b$ parameters in determining the transformation. Such an approach may be desirable when small samples are used.

The results reported here suggest that, in terms of the presence of biased items, the accuracy of the linking transformation is a function of the mumber of biased items in the test rather than the number of unbiased items or the percentage of items that are biased. While some minimum number of unbiased items is needed to determine the linking transformation, the findings of this study indicate that it is the size of the minority of biased items rather than the size of the majority of unbiased itenns that has the most profound effect on the linking transformation.

The presence of large numbers of biased items appears to increase false alarm rates more than it lowers hit rates. McCauley and Mendoza (1985) have reported similar findings from their simulation sudy of item bias. Therefore, the primary concern with using a noniterative procedure for linking metrics and identifying item bias would be that many of the irems labeled as biased by the noniterative approach may in fact be unbiased, rather than the concern that truly biased items will not be identified.

Finally, it should be emphasized that the results reported here may not generalize to one-parameter (Rasch) or three-parameter item response models. Further research is needed to determine the effectiveness of the iterative linking procedure when one- or three-parameter models are used.

\section{Correlessions}

Investigators of item bias using the two-parameter IRT model are encouraged to use iterative linking. The iterative procedure improved item bias detection and classification over the noniterative approach in each of the conditions examined in this study. The improvement was most signifcant when large numbers of biased items were present. Implementation of the iterative procedure is relatively simple and the resulting gains in item bias assessment may be substantial. 


\section{苚eferences}

Bejar, I., \& Wingersky, M. (1981). An application of item response theory to equating the Test of Standard Written English (College Board Report No. 81-8/ETS No. 81-35). Princelon NJ: Educational Testing Service.

Bimbaum, M. (1968). Some latent trait models and their use in inferring an examinee's ability. In F. M. Lord \&. R. Novick, Statistical theories of mental test scores (pp. 397-472). Reading MA: Addison-Wesley.

Bock, R. D., \& Lieberman, M. (1970). Fitting a response model for nd dichotomously scored items. Psychometrika, 35, 179-197.

Candell, G. L., \& Hulin, C. L. (1986). Cross-language and cross-cultural comparisons: Independent sources of information about item non-equivalence. Joumal of Cross-Cultural Psychology, 17, 417-440.

Divgi, D. R. (1985). A minimum chi-square method for developing a common metric in item response theory. Applied Psychological Measurement, 9, 413-415.

Drasgow, F. (1987). Study of the measurement bias of two standardized psychological tests. Joumal of Applied Psychology, 72, 19-29.

Drasgow, $\mathbb{F}$. (in press). An evaluation of marginal maximum likelihood estimation for the two-parameter logistic item response model. Applied Psychological Measurement.

Haebara, T. (1980). Equating logistic ability scales by a weighted least squares method. Japanese Psychological Research, 22, 144-149.

Hulin, C. L., Lissak, R. I., \& Drasgow, F. (1982). Recovery of two- and three-parameter logistic item characteristic curves: A monte carlo study. Applied Psychological Measurement, 6, 249-260.

Hulin, C. L., \& Mayer, L. (1986). Psychometric equivalence of a translation of the job descriptive index into Hebrew. Joumal of Applied Psychology, 71, 83-94.

Kok, F. G., Mellenbergh, G. J., \& van der Flier, $H$. (1985). Detecting experimentally induced item bias using the iterative logit method. Journal of Educational Measurement, 22, 295-303.
Linn, R. L., Levine, M. V., Hastings, C. N., \& Wardrop, I. L. (1981). Item bias in a test of reading comprehension. Applied Psychological Measurement, 5, $159-173$.

Lord, $\mathrm{F}$. M. (1980). Applications of item response theory to practical testing problems. Hillsdale $\mathrm{NJ}$ : Erlbaum.

Marco, G. (1977). Item characteristic curve solutions to three intractable testing problems. Journal of Educational Measurement, 14, 139-160.

McCauley, C. D., \& Mendoza, J. (1985). A simulation study of item bias using a two-parameter item response model. Applied Psychological Measurement, 9, 389400.

Segall, D. O. (1983). Test characteristic curves, item bias, and transformation to a common metric in item response theory: A methodological artifact with serious consequences and a simple solution. Unpublished manuscript, University of Illinois, Department of Psychology.

Shepard, L., Camilli, G., \& Williams, D. M. (1984). Accounting for statistical anifacts in item bias research. Joumal of Educational Statistics, 9, 93-128.

Stocking, M. L., \& Lord, F. M. (1983). Developing a common metric in item response theory. Applied Psychological Measurement, 7, 201-210.

Vale, C. D., Maurelli, V. A., Gialluca, K. A., Weiss, D. J., \& Ree, M. J. (1981). Methods for linking item parameters (AFHRL-TR-81-10). Brooks Air Force Base TX: U.S. Air Force Human Resources Laboratory.

van der Flier, H., Mellembergh, G. J., Ader, H. I., \& Wijn, M. (1984). An iterative item bias detection method. Journal of Educational Measurement, 21, $131-145$.

\section{A}

Send requests for reprints or further information to Gregory Candell, CTB/McGraw-Hill, 2500 Garden Road, Monterey CA 93940, U.S.A. 